\title{
Design of Optimal Beamforming using Particles Swarm Optimization
}

\author{
Mohammad K. Khawaldeh \\ Department of Control and Communication system, \\ National Electrical Power Company \\ Amman, Jordan
}

\author{
Saed Khawaldeh \\ Erasmus+ Joint Master Program in Medical Imaging \\ and Applications; University of Burgundy (France), \\ UNICLAM (Italy) and University of Girona (Spain)
}

\begin{abstract}
Beamforming is a signal processing technique by which an array of receivers sensitive to signals from all directions can be processed to form one larger more sensitive receiver that can identify which direction signals originate. In this paper the narrowband beamforming and wideband beamforming using Tapped delay line will be investigated. Some applications in wireless communication systems need to design beamformer with a specified response like a sidelobe level less than threshold level or forming a null response points in interference direction or jamming frequency. So we need to design optimal beamforming using optimization technique, to achieve a desired response. In this paper the common global optimization technique like Particle Swarm Optimization (PSO) will be discussed and will used to design a different examples on narrowband and wideband beamforming.
\end{abstract}

\section{General Terms}

Signal Processing, Wireless Communications, Optimization.

\section{Keywords}

Wideband Beamforming, Tapped Delay Line Filter, Particle Swarm Optimization.

\section{INTRODUCTION}

Telephony, data, image, Internet and video applications are essential to future wireless communication systems. Multimedia communications, as mentioned above, will demand more efficient antenna array structures than traditional monopole antennas.

Array processing techniques like a beamforming help in improving the system performance, such as coverage increase, multipath fading mitigation, handoff simplification, and privacy enhancement. Thus, application of antenna arrays in wireless communications may be considered as one of the most promising ways to accommodate the rapidly growing service demands for multimedia transportation [1-2]. The received signals on an array of sensors are multiplied by a complex weights before summing to produce the array output and directional constraints on array weights [3] are imposed to have a desired response of the array in a given look direction. The constraints protect the desired signal arriving from the look direction when the array weights are obtained by solving some optimization problem. As the signal bandwidth increases the performance of the array system using this narrowband structure, where induced signals are multiplied by complex weights, starts to deteriorate [4].

For processing broadband signals a tapped delay line (TDL) filter in front of each element is used [5-7] and filter coefficients are determined by solving some constrained beamforming problem [8-9]. Many of these constraints are designed to obtain a desired frequency response of the processor in the look direction while simultaneously cancel the unwanted directional sources impinging on the array from other directions.

This paper is organize as follow : a narrowband beamforming and a wideband beamforming using TDL is introduced in section 2 followed by a review of PSO technique and required parameters in section 3 , section 4 contains an optimal beamforming design example using PSO . A brief conclusion is presented in section 5 .

\section{BACKGROUND}

The first and foremost, beamformer can be grouped according to bandwidth of signal environment. This can be either narrowband or broadband, narrowband beamforming is generally less complex. Therefore, the question arises which signals can be considered narrowband, and where broadband characteristics have to be assumed. According to [10], if the ratio between the signal bandwidth and the mid-band frequency falls below a specific threshold, the signal can be considered narrowband. The value of the threshold typically $2.5 \%$-depends upon the application and no fixed standard definition is available. The beamforming structure, shown in Fig.1, works effectively only for narrowband signals like sinusoidal ,Where $M$ sensors sample the wave field spatially and the output $\mathrm{y}(\mathrm{t})$ at time $\mathrm{t}$ is given by an instantaneous linear combination of these spatial samples $\mathrm{x}_{\mathrm{m}}(\mathrm{t}), \mathrm{m}=0,1, \ldots, \mathrm{M}-$ 1 , as equation below:

$$
y(\mathrm{t})=\sum_{m=0}^{M-1} x_{m}(t) w_{m}^{*}
$$

For complex plane wave $\left(\mathrm{e}^{\mathrm{j} \omega t}\right)$ with an angular frequency $\omega$ and a Direction of arrival (DOA) angle $\theta$, where $\theta \in[-\pi / 2$ $\pi / 2]$ is measured with respect to the broadside of the linear array the signal received by the first sensor is $\mathrm{x}_{0}(\mathrm{t})=\mathrm{e}^{\mathrm{j} \omega \mathrm{t}}$ and by the $\mathrm{m}^{\text {th }}$ sensor is $x_{m}(t)=\mathrm{e}^{\mathrm{j} \omega(\mathrm{t}-\mathrm{\tau m})}, \mathrm{m}=1,2, \ldots \ldots, \mathrm{M}-1$, where $\tau_{m}$ is the propagation delay for the signal from sensor 0 to sensor $\mathrm{m}$ and is a function of $\theta$. Then the beamformer output is:

$$
y(\mathrm{t})=e^{\mathrm{j} \omega \mathrm{t}} \sum_{m=0}^{M-1} e^{-\omega \tau \mathrm{m}} w_{m}^{*}
$$

with $\tau_{0}=0$. The response of this beamformer is given by:

$$
\mathrm{P}(\omega, \theta)=\sum_{m=0}^{M-1} e^{-\omega \tau \mathrm{m}} w_{m=w^{H}}^{*} \mathrm{~d}(\omega, \theta)
$$

Where the weight vector $\mathrm{w}$ holds the $\mathrm{M}$ complex conjugate coefficients of the sensors given by: 


$$
w=\left[w_{0} w_{1} \ldots w_{M-1}\right]^{T}
$$

and the vector $d(\omega, \theta)$ is given by:

$$
d(\omega, \theta)=\left[\begin{array}{lll}
1 e^{-j \omega \tau_{1}} & \ldots e^{-j \omega \tau} M-1
\end{array}\right]
$$

We refer to $d(\theta, \omega)$ as the array response vector, which is also known as the steering vector or

Direction vector.

For a uniformly spaced linear array with an inter-element spacing $d$, also $\tau_{m}=m \tau_{1}=m(d \sin \theta) / c$ and $\omega \tau_{m}=m(2 \pi d \sin$ $\theta) / \lambda$. we set $\boldsymbol{d}=\lambda \boldsymbol{\Omega}$, then $\boldsymbol{\omega} \boldsymbol{\tau}_{\boldsymbol{m}}=\boldsymbol{m} \boldsymbol{\pi} \sin \boldsymbol{\theta}$ and the response of the uniformly spaced narrowband beamformer is given by:

$$
P(\omega, \theta)=\sum_{m=0}^{M-1} e^{-j m \pi \sin \theta} w_{m}^{*}
$$

The narrowband beamforming structure, shown in Fig.1, works effectively only for narrowband signals. When the signal bandwidth increases, its performance will degrade significantly. For wideband signals, since each of them consists of infinite number of different frequency components, the value of the weights should be different for different frequencies. This is why the narrowband beamforming structure with a single constant coefficient for each received sensor signal will not work effectively in a wideband environment. The frequency dependent weights can be achieved by sensor delay-lines (SDLs). Traditionally, an easy way to form such a set of frequency dependent weights is to use a series of tapped delay-lines (TDLs) or FIR (Finite impulse response), IIR (infinite impulse response) filters in its discrete form. Both TDLs and FIR/IIR filters perform a temporal filtering process to form a frequency dependent response for each of the received wideband sensor signals to compensate the phase difference for different frequency components. Such a structure is shown in Fig.2 [11].

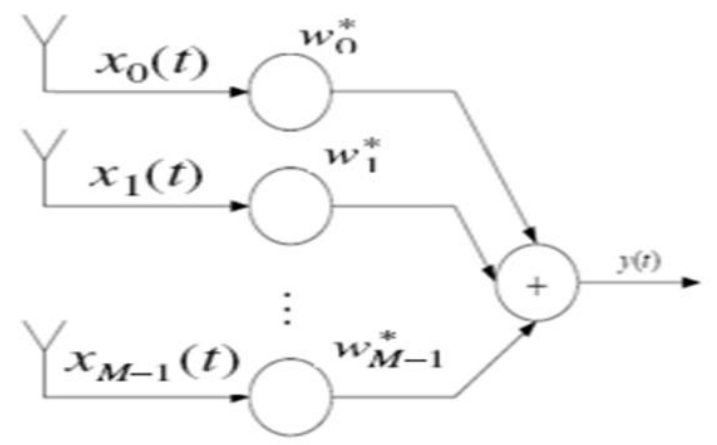

Fig.1: Narrowband beamforming

The beamformer obeying this architecture samples the propagating wave field in both space and time. The output of such a wideband beamformer can be expressed as:

$$
y(t)=\sum_{m=0}^{M-1} \sum_{i=0}^{\mathrm{J}-1} \mathrm{x}_{\mathrm{m}}\left(\mathrm{t}-\mathrm{iT}_{\mathrm{s}}\right) \times \mathrm{w}_{\mathrm{m}, \mathrm{i}}^{*}
$$

where $x_{m}(t)$ is the receive signal at antenna element $\boldsymbol{m}, \boldsymbol{w}$ is weight value, $\mathrm{J}-1$ is the number of delay elements associated with each of the M sensor channels in Fig.2 and Ts is the delay between adjacent taps of the TDLs. We now analyze the array's response to an impinging complex plane wave $\left(\mathrm{e}^{\mathrm{j} \omega \mathrm{t}}\right)$ with an angular frequency $\omega$ and a DOA angle $\theta$, where $\theta \in\left[\begin{array}{ll}-\pi / 2 & \pi / 2\end{array}\right]$ is measured with respect to the broadside of the linear array. Then the signal received by the first sensor is $\mathrm{x}_{0}(\mathrm{t})=\mathrm{e}^{\mathrm{j} \omega \mathrm{t}}$ and by the $\mathrm{m}^{\text {th }}$ sensor is $x_{m}(t)=$ $\mathrm{e}^{\mathrm{j} \omega(\mathrm{t}-\tau \mathrm{m})}$, then we have $\boldsymbol{x}_{\boldsymbol{m}}\left(\boldsymbol{t}-\boldsymbol{i} \boldsymbol{T}_{s}\right)=\boldsymbol{e}^{\boldsymbol{j} \boldsymbol{\omega}\left(\boldsymbol{t}-\left(\boldsymbol{\tau}_{m+} \boldsymbol{i} \boldsymbol{T}_{s}\right)\right)}, \mathrm{m}=$ $1,2, \ldots \ldots, \mathrm{M}-1, \mathrm{i}=0, \ldots, \mathrm{J}-1$. The array output is given by equation below:

$$
y(t)=e^{j \omega t} \sum_{m=0}^{M-1} \sum_{i=0}^{J-1} e^{-j \omega\left(\tau_{m}+i T_{s}\right)} \times w_{m, i}^{*}=e^{j \omega t} \times P(\omega, \theta)
$$

where $\tau_{m}$ is the propagation delay for the signal from sensor 0 to sensor $\mathbf{m}$ and is a function of $\theta$ For a uniformly spaced linear array with an inter-element spacing $d$. We have $\tau_{m}=$ $m \tau_{1}=m(d \sin \theta) / c$ and $\omega \tau_{m}=m(2 \pi d \sin \theta) / \lambda$. To avoid aliasing, $d \leq \lambda_{\min } / 2$ where $\lambda_{\min }$ is the wavelength of the signal component with the highest frequency $\omega_{\max }$. In its discrete form, $\boldsymbol{T}_{\boldsymbol{s}}$ is the temporal sampling period of the system and should be no more than half the period $\mathrm{T}_{\min }$ of the signal component with the highest frequency according to the Nyquist sampling theorem, i.e. $T_{s} \leq \mathrm{T}_{\min } / 2$. The $\mathrm{P}(\omega, \theta)$ is the beamformer's frequency and angle dependent response. It can be expressed in as shown in equation below:

$$
P(\omega, \theta)=\sum_{m=0}^{M-1} \sum_{i=0}^{J-1} e^{-j \omega\left(\tau_{m}+i T_{s}\right)} \times w_{m, i}^{*}
$$

With the normalized frequency $\Psi=\omega T_{S}, \omega\left(m \tau_{l}+i T_{S}\right)$ changes to $m \mu \Psi \sin \theta+i \Psi$ with $\mu=d /\left(c T_{S}\right)$, we have (Zaman at al., 2011):

$$
\begin{gathered}
\mathrm{P}(\omega, \theta)=\sum_{\mathrm{m}=0}^{\mathrm{M}-1} \sum_{\mathrm{i}=0}^{\mathrm{J}-1} \mathrm{e}^{-\mathrm{j} \Psi(\mathrm{m} \mu \sin (\theta)+1)} \times \mathrm{w}_{\mathrm{m}, \mathrm{i}}^{*} \\
\mathrm{P}(\mathrm{f}, \theta)=\sum_{m=0}^{M-1} \sum_{i=0}^{J-1} e^{-j \pi \frac{f}{f_{\max }}(m \mu \sin (\theta)+1)} \times w_{m, i}^{*}
\end{gathered}
$$

After we define a response of narrowband beamforming and wideband beamforming in equations above, the statement of the current problem (design optimal beamforming) simply reduces to use the PSO algorithm to find the weights vector , $\mathrm{w}$, of the array elements that result an beamforming response with minimum SLL and, if desired nulls at specific directions and frequency. 


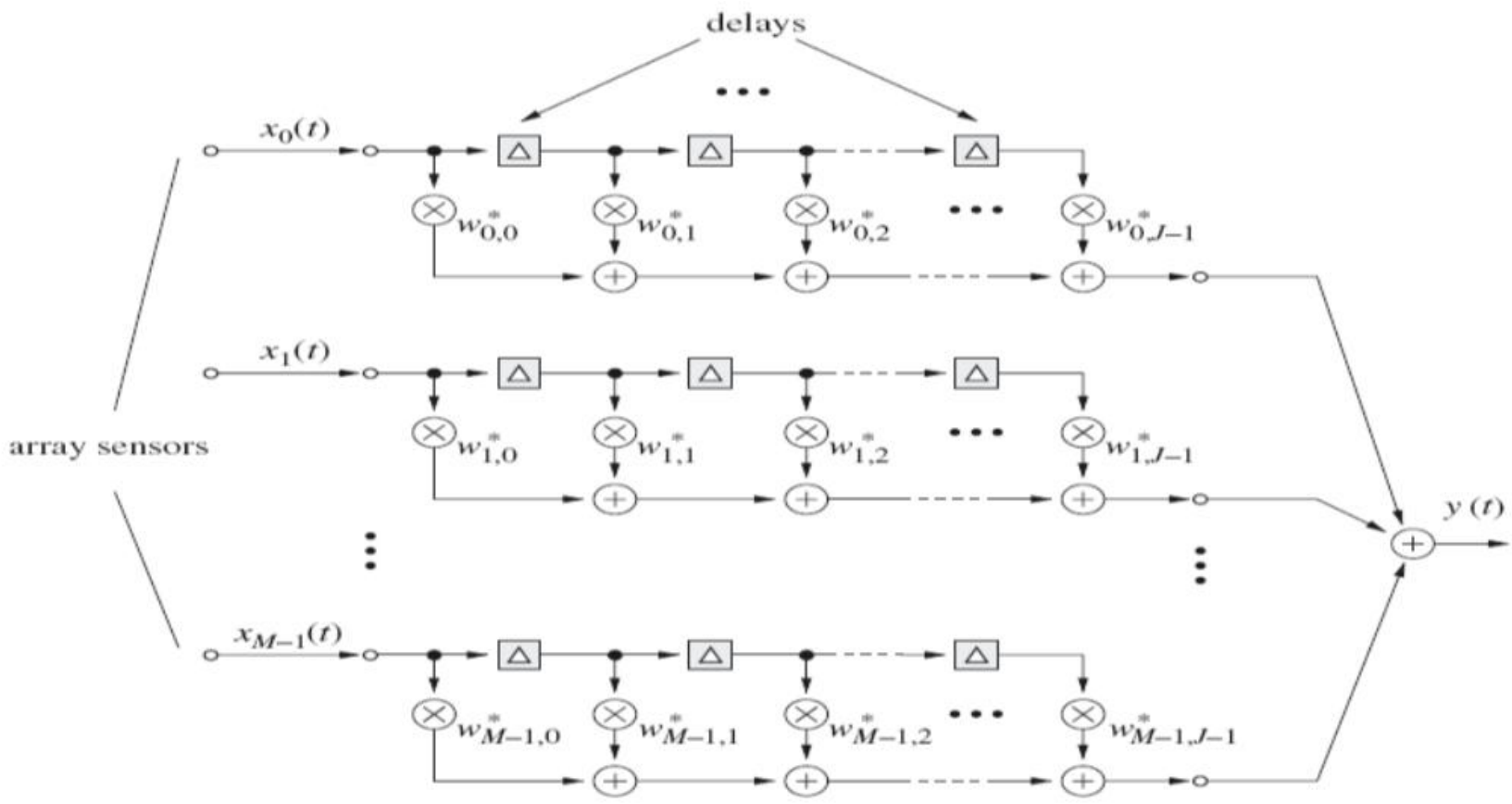

Fig.2: Wideband beamforming structure

\section{PARTICLE SWARM OPTIMIZATION TECHNIQUE (PSO)}

Optimization simply means finding a "better" solution to a problem. It is the process of adjusting inputs of a phenomenon to obtain a desirable output. Most of the real-life problems are optimization problems, indeed. Scientists, engineers, manufacturer's financiers and even sociologists consult with different types of optimization techniques in order to obtain efficient solutions to their problems. In mathematics, optimization deals with seeking for the minima or maxima of a function within a search space.

Particle swarm optimization is one of the famous global optimization techniques which is inspired by social behaviour of "swarms" in the nature. The swarms like bird flock, bees swarm and fish school are very successful in finding food in nature. They are in continuous interaction with each other while searching for food in a large area. Each bird or fish tells the location and quantity of the food that it found to the others. Thus, by taking into account the location and quantity data from each member of the swarm, whole swarm tends towards the optimum location where maximum amount of food is present [12].

The particle swarm optimization technique was first introduced in [13], by James Kennedy who is a social psychologist; and by Russell Eberhart who is an electrical engineer. In their articles "Particle Swarm Optimization" and "A new optimizer using particle swarm theory", they described the stages of development of their technique from a social study of swarms to an optimizer. They also discussed the basic concepts of this technique and the results obtained from applications upon which their technique performed successfully. Particle swarm optimization technique has been further developed by many researchers and has undergone many changes since its introduction in 1995. According to Kennedy and Eberhart, particle swarm optimization can be used in solution of multidimensional nonlinear global optimization problems effectively. Moreover, it has a very simple concept and can be implemented by using primitive mathematical operators which make the technique computationally inexpensive in terms of both memory and speed requirements. The operation of PSO is summarized in flowchart shown in Fig.3.

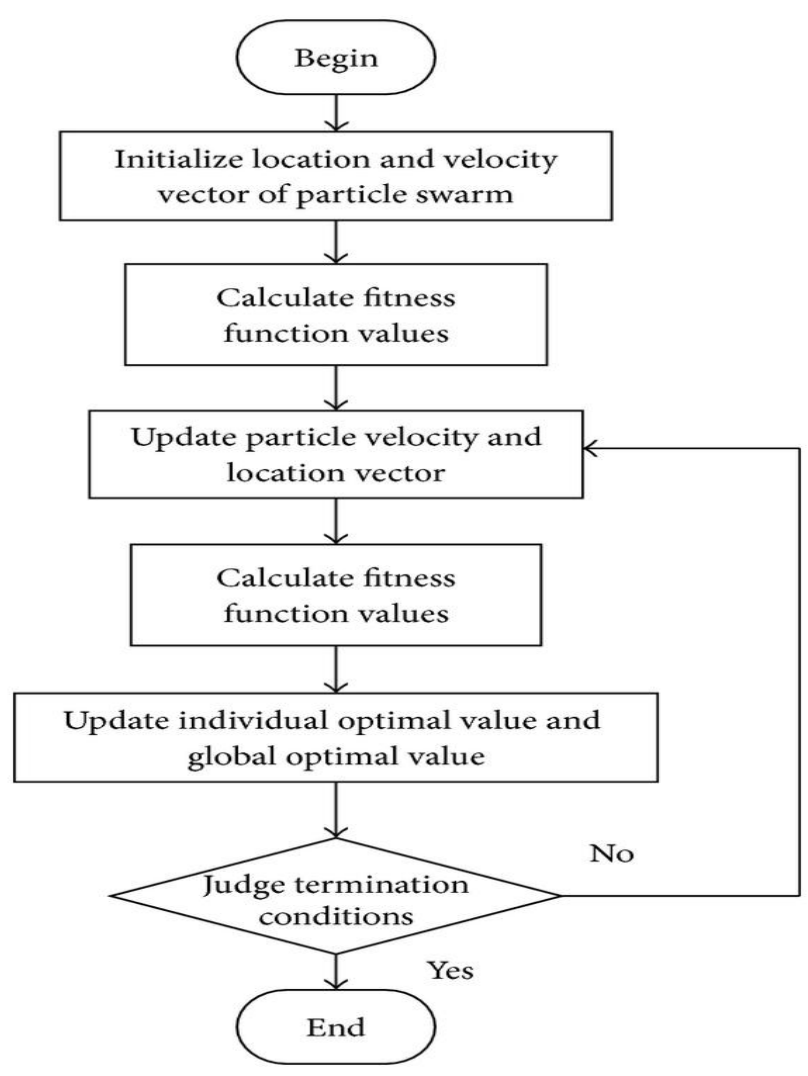

Fig.3: Flowchart of main steps for PSO algorithm 
In a flowchart diagram. After defining the solution space and the fitness function, the PSO algorithm starts by randomly initializing the position and velocity of each particle in the swarm. For an $\mathrm{N}$ dimensional problem, the position and velocity can be specified by an $\mathrm{Mo} \times \mathrm{N}$ matrices as follows:

$$
\begin{aligned}
X & =\left[\begin{array}{cccc}
x_{11} & x_{12} & \cdots & x_{1 N} \\
x_{21} & x_{22} & \cdots & x_{2 N} \\
\vdots & & & \\
x_{M o 1} & x_{M o 2} & \cdots & x_{M o N}
\end{array}\right] \\
V & =\left[\begin{array}{cccc}
v_{11} & v_{12} & \cdots & v_{1 N} \\
v_{21} & v_{22} & \cdots & v_{2 N} \\
\vdots & & & \\
v_{M o 1} & v_{M 2} & \cdots & v_{M o N}
\end{array}\right]
\end{aligned}
$$

Where $M o$ is the number of particles in the swarm. Each row of the position matrix represents a possible solution to the optimization problem. The velocity of each particle depends on the distance of the current position to the positions that resulted in good fitness values. To update the velocity matrix at each iteration, every particle should know its personal best and the global best position vectors. The personal best position vector defines the position at which each particle attained its best fitness value up to the present iteration. The personal best positions can be defined by the matrix below.

$$
P=\left[\begin{array}{ccl}
P_{11} & P_{12} & P_{1 N} \\
P_{21} & P_{22} & P_{2 N} \\
\vdots & \ldots & \vdots \\
P_{M o 1} & P_{M 2} & P_{M o N}
\end{array}\right]
$$

The global best position vector defines the position in the solution space at which the best fitness value was achieved by all particles, and is defined by matrix below.

$$
G=\left[g_{1} g_{2} \ldots g_{N}\right]
$$

All the information needed by the PSO algorithm, Fig.3, is contained in $\mathrm{X}, \mathrm{V}, \mathrm{P}$, and $\mathrm{G}$. The core of the PSO algorithm is the method by which these matrices are updated in every iteration of the algorithm. These matrices are updated such that each particle takes the path of a damped oscillatory movement toward its personal best and the global best positions in every new iteration. To achieve this, the velocity matrix is updated according to [14-17] as:

$$
v_{m n^{k}=} w_{o^{v}} m n^{k-1}+c 1 U_{n 1^{k}}\left(P m n^{k-x} m n^{k-1}\right)+c 2 U_{n 2^{k}}\left(g n^{k-x} m n^{k-1}\right)
$$

where $\mathrm{k}$ and $\mathrm{k}-1$ the superscripts and refer to the time index of the current and the previous iterations, $\mathrm{U} 1$ and $\mathrm{U} 2$ are two uniformly distributed random numbers in the interval $[0,1]$ and these random numbers are different for each $n$ of the components of the particle's velocity vector. The parameter c1 and c2 are self-confidence and swarm confidence respectively, it specify the relative weight of the personal best position versus the global best position. The value of these parameter are very important in speed of convergence for optimization problem then enhancement it performance. The value of these parameter depending in references and search paper [18] can be equal 2. Or can be equal 1.49 [19].
Regarding the parameter $\left(\mathrm{w}_{\mathrm{o}}\right)$ inertia weight, it determines how the previous velocity of the particle influences the velocity in the next iteration how far the particle is from its personal best and global best positions. According to several studied the value of inertia weight $\mathrm{w}$ can be a constant $\mathrm{wo}=1$, 0.79 ; [19], also wo can be linearly damped function with iterations starting at 0.9 and decreasing linearly to 0.4 at the last iteration [12], or depending on [20], wo can be exponentially decay function starting at 0.9 and decreasing to 0.4 at last iteration. After we define the required updating velocity parameters, the position matrix is updated at each iteration according to:

$$
X^{k}=X^{k-1}+V^{k}
$$

The PSO algorithm, like other evolutionary algorithms, uses the concept of fitness to guide the particles during their search for the optimum position vector in the $\mathrm{N}$ dimensional space. The fitness defines how well the position vector of each particle satisfies the requirements of the optimization problem. The PSO at each iteration define the best particle position which has a best fitness, and pass this information to all particle to adjusting their velocity and position vector. The core parameter in all optimization algorithm is defined the requirement of optimization problem or answer a question what is the main purpose for the optimization problem. The answer of this question is fitness function which is translate the optimization purpose to mathematical equation. In design an optimal beamforming, there are many variable (objective variable) can be used by optimization algorithm to achieve desired response or evaluate the fitness like weights set displacement between antenna elements. Depending on the application in this paper, we will use the weights set with minimum sidelobe level forming null in specific points. To achieve this goal we used the following fitness functions:

\section{1- For a narrowband beamforming}

FitnessFunction $=\operatorname{Min}\left[O F 1 \times \operatorname{Max}\left[\left|P\left(\theta_{S}\right)\right|\right]+O F 2 \times \sum_{K=1}^{K_{0}}\left|P\left(\theta_{K}\right)\right|\right]$

2- $\quad$ For a wideband beamforming

FitnessFunction $=\operatorname{Min}\left[O F 1 \times \operatorname{Max}\left[\left|P\left(f, \theta_{S}\right)\right|\right]+O F 2 \times \sum_{K=1}^{K_{0}}\left|P\left(f_{K}, \theta_{K}\right)\right|\right]$

where OF1, OF2 are optimization factors (important in optimization problem) for sidelobe and null points respectively, $\left|\mathrm{P}\left(\theta_{\mathrm{S}}\right)\right|$ : magnitude of narrowband response at $\left(\theta_{\mathrm{S}}\right) \quad\left|\mathrm{P}\left(\theta_{\mathrm{K}}\right)\right|$ : is the magnitude of null points, $\left|\mathrm{P}\left(\mathrm{f}, \theta_{\mathrm{S}}\right)\right|$ : magnitude of wideband response at $\left(f, \theta_{S}\right), f$ is frequency range $\left[f_{l}, f_{u}\right]$ Where $f_{l}, f_{u}$ are lower and upper limit for frequency range respectively, $\theta_{\mathrm{S}}$ is a range of sidelobes direction $=\left[\theta_{\mathrm{l}}, \theta_{\mathrm{u}}\right]_{\text {left }}\left[\theta_{\mathrm{l}}, \theta_{\mathrm{u}}\right]_{\text {right }}$, Where $\theta_{\mathrm{l}}, \theta_{\mathrm{u}}$ are lower and upper limit for sidelobe respectively. $\left|\mathrm{P}\left(\mathrm{f}_{\mathrm{k}}, \theta_{\mathrm{K}}\right)\right|$ : is the magnitude of null points, $\theta_{\mathrm{K}}$ : the directions of null points , $\mathrm{f}_{\mathrm{k}}$ : the frequency of null points, $\mathrm{k} 0$ : number of null points

\section{EXPERIMENTATS AND RESULTS}

The PSO algorithm outlined in the previous section is illustrated by the following design examples. The PSO algorithm was implemented using MATLAB. The first design example is design a narrowband beamforming with sidelobe level in band $\left[-90^{\circ},-17^{\circ}\right]$ and $\left[17^{\circ}, 90^{\circ}\right]$ less than $-30 \mathrm{~dB}$, in this example, the array is composed of 10 identical isotropic elements, in PSO algorithm the number of particle in swarm Mo was chosen equal 20, the number of iteration was chosen large enough to guarantee the convergence PSO to desired solution, initial solution is ones and inertia weight $\left(\mathrm{w}_{\mathrm{o}}\right)$ is linear damped from 0.9 to 0.4 also $c_{1}, c_{2}$ equal 2. Fig.4 and 
Fig.5, shown the output from PSO algorithm. We can see, the response of design approximately symmetrical with a desired response with the max sidelobes level are less than $-30 \mathrm{db}$ and it was achieved within 250 iterations. In Fig.4, we compared between the initial response (weights are ones) and PSO output response, we can see that, the different in sidelobes level approximately $10 \mathrm{~dB}$, but the cost is the main beamwidth increase a few degrees. At each iteration the velocity of each particles in swarm change in magnitude and dimension,so the Fig.6, is shown a magnitude of velocities $\mathrm{V}$ for the best particle, as we see in Fig.6 the velocity of each element have random variation till to reach 0 value within 250 iteration which mean that the particle didn't change its value after this point, Because it reach to the global best solution.

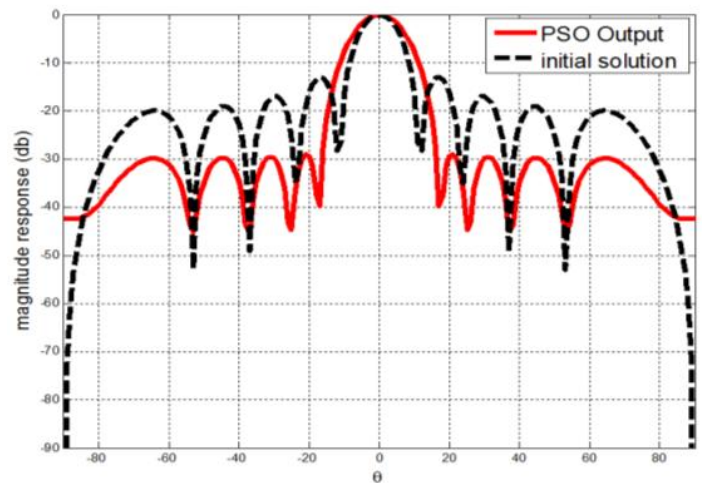

Fig.4: Normalized magnitude response of optimal narrowband beamformer with max sidelobe level -30db

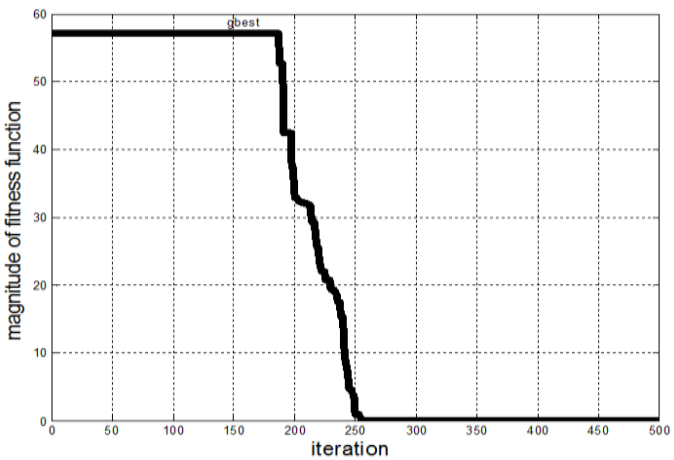

Fig.5: Convergence curve of the fitness function versus the number of iterations
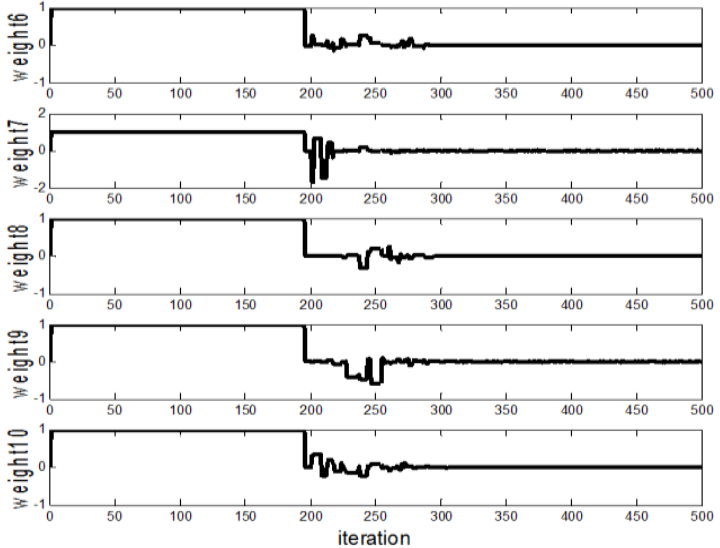

Fig.6: Convergence of a best particle velocities versus the number of iterations
The second design example is design a narrowband bamfomring with null points at direction $\theta=-70,-50,40,60$ and a sidelobes in band $\left[-90^{\circ},-17^{\circ}\right]$ and $\left[17^{\circ}, 90^{\circ}\right]$.We used the same parameters in previous example only we changed the initial solution to become random values, the optimization factors are $\mathrm{OF} 1=10$, $\mathrm{OF} 2=$ $\left(50\left(\theta=-70^{\circ}\right), 40\left(\theta=-50^{\circ}\right), 40\left(\theta=40^{\circ}\right), 50\left(\theta=50^{\circ}\right)\right)$. The results of this design were plotted in Fig.7 and Fig.8, the desired null points are achieved with average response equal $90 \mathrm{~dB}$ within 500 iteration.

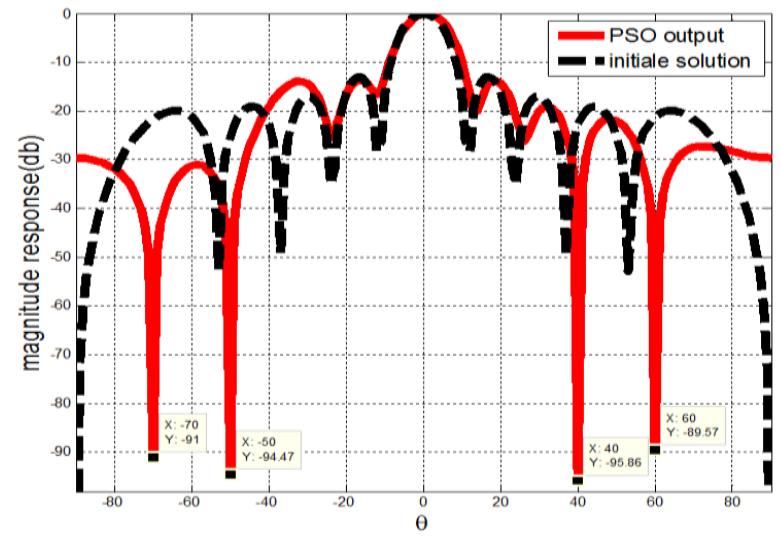

Fig.7: Normalized magnitude response of a narrowband beamforming with a null points at direction $\theta_{K}=-7^{\circ}$,$50^{\circ}, \mathbf{4 0}^{\circ}, \mathbf{6 0}^{\circ}$. With initial solution is random complex values

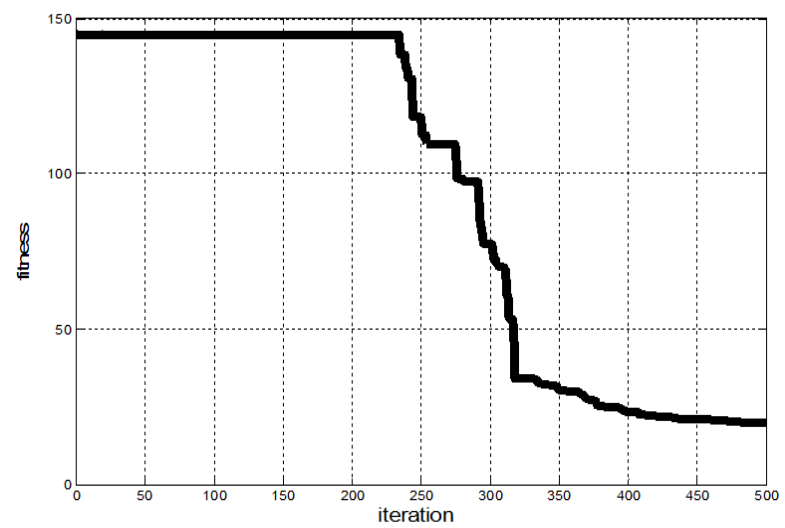

Fig.8: Convergence curve of the fitness function versus the number of iterations

Third example in narrowband beamforming is design a narrowband beamformer with a response has a broad null band from the direction $\theta=-58^{\circ}$ to $\theta=-63^{\circ}$, We used the same parameters in previous example only We changed optimization factor to $\mathrm{OF} 1=10, \mathrm{OF} 2=(40$ for the band $(\theta=-$ $\left.58^{\circ}\right)$ : $\left.\left(\theta=-63^{\circ}\right)\right)$.

The output and result are plotted in Fig.9 and Fig.10, we can see the desired response abroad null point with width $6^{\circ}$ degree is achieved with average response equal $-70 \mathrm{~dB}$ within 500 iterations. 


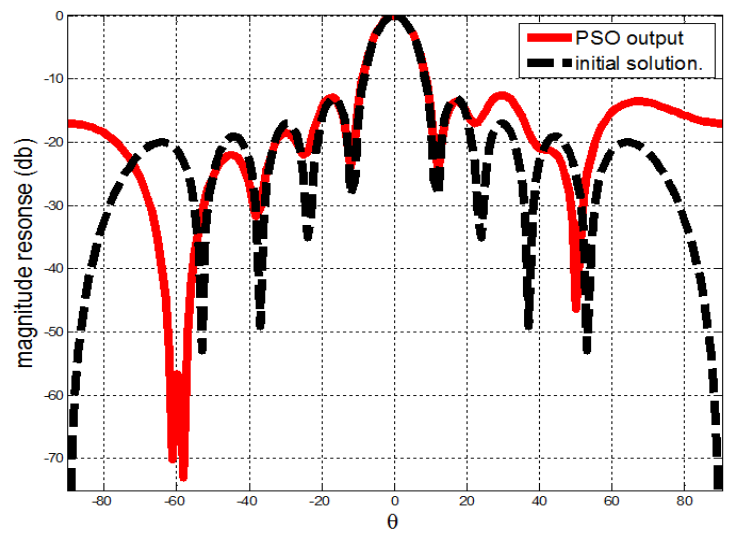

Fig.9: Normalized magnitude response of a narrowband beamforming with a broad null band at direction $\theta_{\mathrm{K}}=$ $58^{\circ}:-63^{\circ}$. With initial solution is random complex values

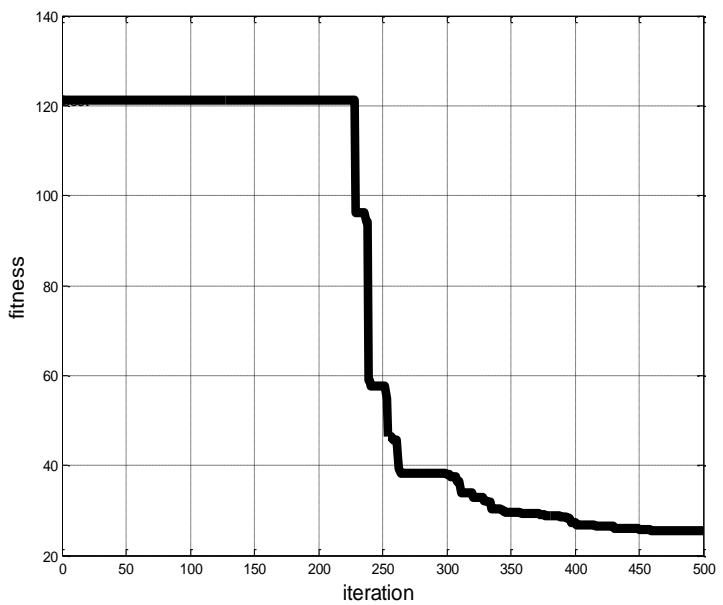

Fig.10:.Convergence curve of the fitness function versus the number of iterations

For wideband beamforming case, the number of particle in swarm Mo was chosen equal 20 and The number of iteration was chosen large enough to guarantee the convergence PSO to desired solution also, We used the frequency range for all design examples is $\mathrm{f}=[1.05 \mathrm{Ghz}-1.45 \mathrm{Ghz}]$.The system parameters are the same for all design, the array is composed of 15 identical isotropic elements, each feeding a 21-tap delay element. The first example illustrate the wideband beamformer with a sidelobes in band $\left[-90^{\circ},-20^{\circ}\right]$ and $\left[20^{\circ}, 90^{\circ}\right]$ and single null point with initial solution is ones and inertia weight $\left(\mathrm{w}_{\mathrm{o}}\right)$ is linear damped from 0.9 to 0.4 also $\mathrm{c}_{1}, \mathrm{c}_{2}$ equal 2. Fig.11, Fig.12 and Fig.13, shown the output from PSO algorithm at direction $-41^{\circ}$ and frequency $\mathrm{f}=1.05 \mathrm{GHz}$, We used optimization factors $\mathrm{OF} 1, \mathrm{OF} 2=5,30$ respectively. We can see from Fig.11, the desired null point is achieved with magnitude equal $-70 \mathrm{db}$ and the desired response need only 100 iteration.

Second design example, We discuss the wideband beamforming with magnitude response have a duple null points at $\left(\theta=-41^{\circ}, \mathrm{f}=1.25 \mathrm{Ghz}\right)$ and $\left(\theta=59^{\circ}, \mathrm{f}=1.05 \mathrm{Ghz}\right), \mathrm{We}$ used the initial solution is complex values with phase equal $30^{\circ}$, for inertia weight $\left(\mathrm{w}_{\mathrm{o}}\right)$ we used the exponential decay function start from 0.9 to 0.4 . and $\mathrm{c}_{1}, \mathrm{c}_{2}$ equal 1.49 and for optimization factor the values are (for sidelobes $\mathrm{OF} 1=10$, for the null point at $\left(\theta=-41^{\circ}, \mathrm{f}=1.25 \mathrm{Ghz}\right) \mathrm{OF}=140$, and at the second null point $\mathrm{OF}=200$ ), Fig.14 and Fig.15, shown the results of desired design and the output of PSO algorithm . The desired response achieved within 100 iterations with null points average magnitude equal $-41 \mathrm{~dB}$.

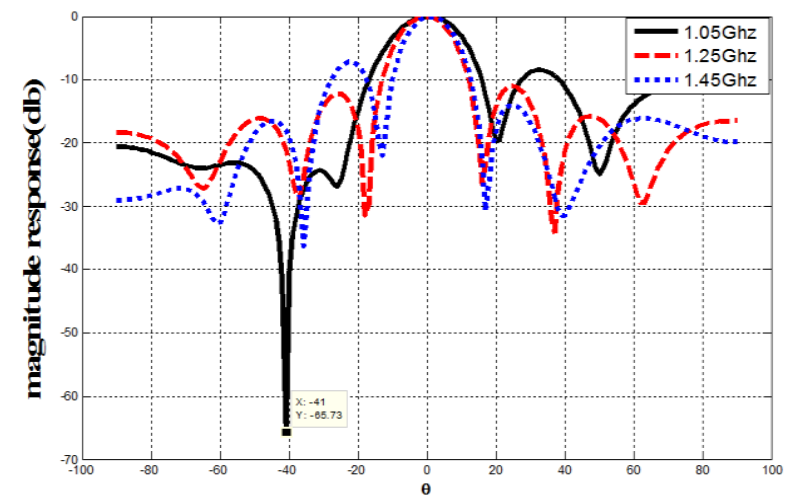

Fig. 11: The magnitude response of wideband beamforming with null point at $\theta=-41^{\circ}, f=1.05 \mathrm{GHz}$

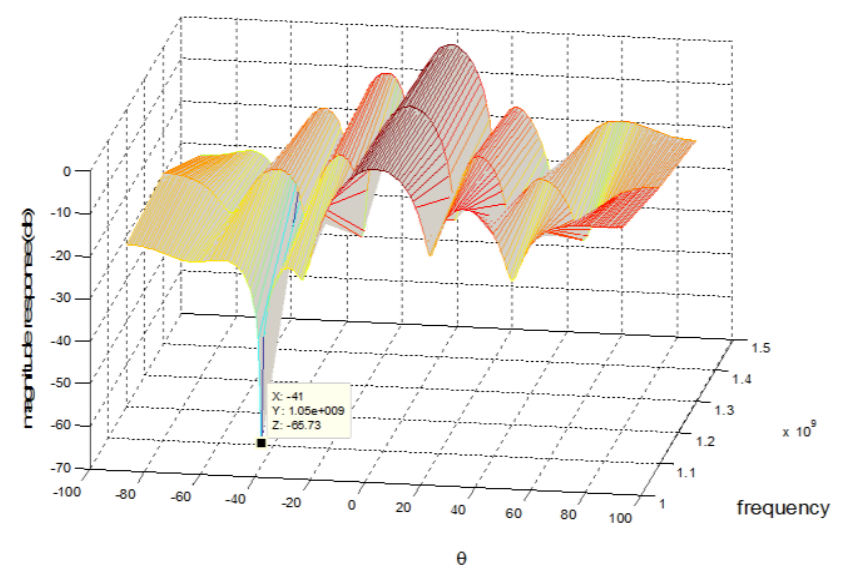

Fig. 12: A 3-D wideband beam pattern example based on an equally spaced linear array with null point $\theta=-41^{\circ}$, $\mathrm{f}=1.05 \mathrm{GHz}, N=15, J=21$ and initial weight=ones

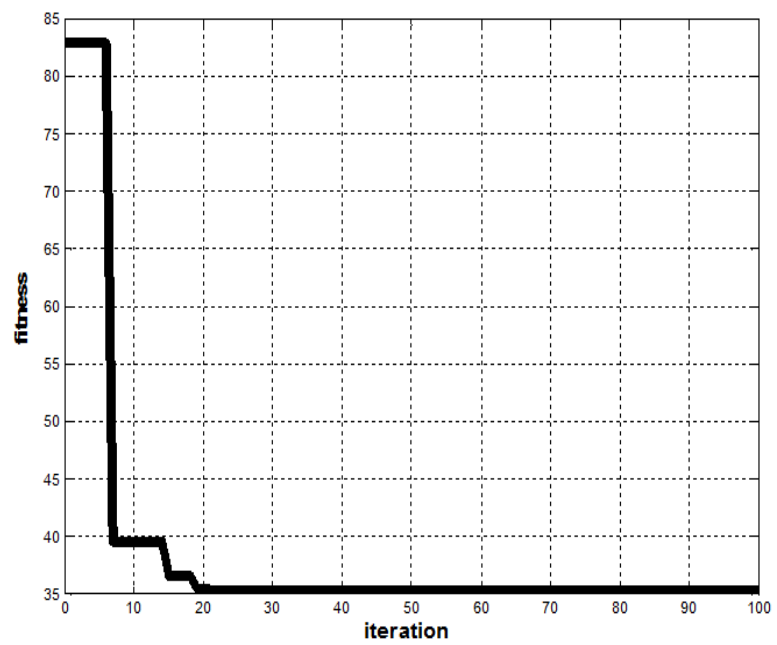

Fig. 13: Convergence curve of the fitness function versus the number of iterations 


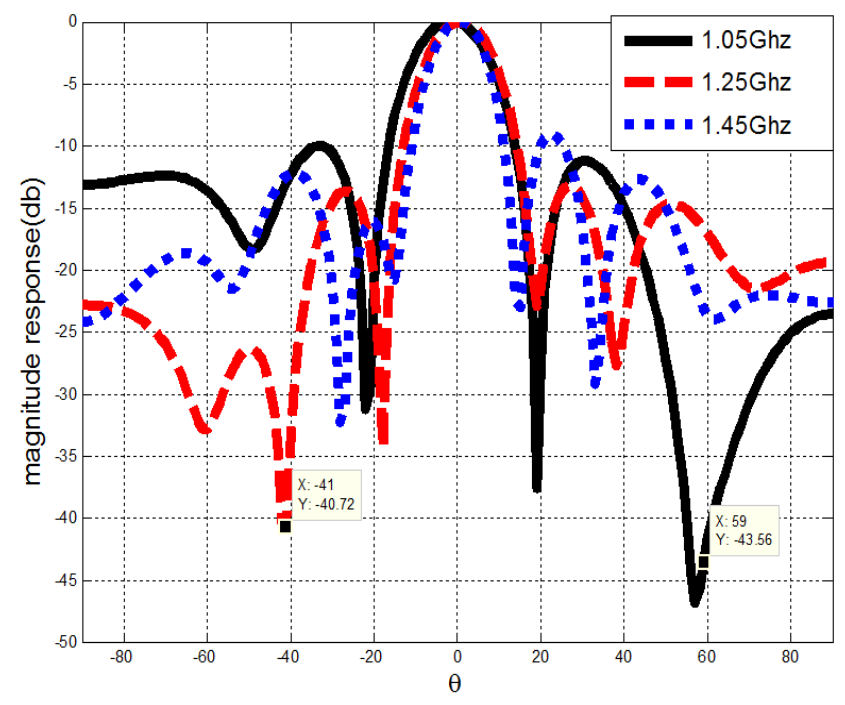

Fig.14: The magnitude response of wideband beamforming with null points at $\theta=59^{\circ} \mathrm{f}=1.05 \mathrm{GHz}$. And $\theta=-41^{\circ}, f=1.25 \mathrm{GHz}$

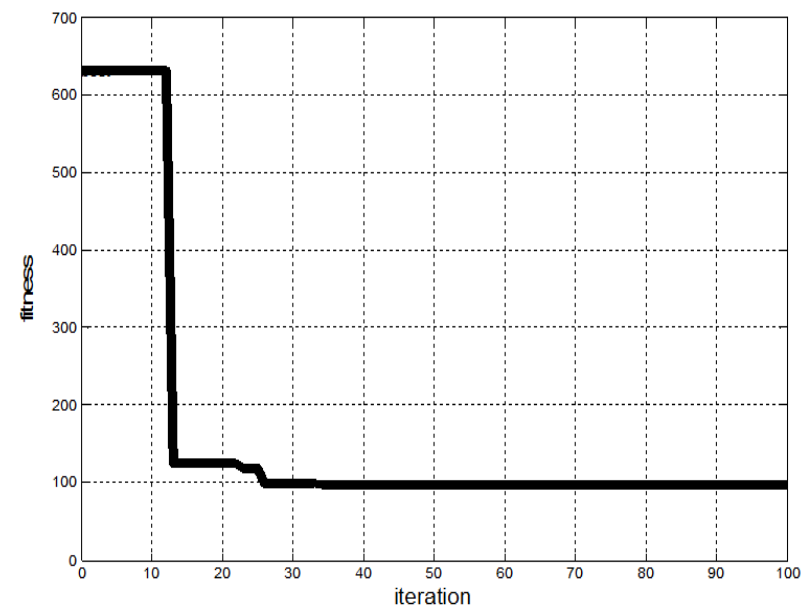

Fig.15: Convergence curve of the fitness function versus the number of iterations

Last example, We designed an wideband beamforming with a magnitude response have a three null points at same direction $\theta=-51^{\circ}$ but different frequencies $(\mathrm{f} 1=1.05 \mathrm{Ghz}, \mathrm{f} 2=1.25 \mathrm{Ghz}$ , f3 $=1.45 \mathrm{Ghz}$ ), in this design we used the same system parameters but we used the initial solution equal to ones, and inertia weight (wo) is linear decay function from 0.8 to 0.4 , and for $\mathrm{c} 1, \mathrm{c} 2$ equal 1.49 and for optimization factors we used the following values (for sidelobe $\mathrm{OF} 1=5$, for the first null point at $\theta=-51^{\circ}, \mathrm{f}=1.05 \mathrm{Ghz} \mathrm{OF}=30$, for second null at $\theta=-51^{\circ}$ ,f $=1.25 \mathrm{Ghz} \mathrm{OF}=45$, and at third null the $\mathrm{OF}=30$ ). Fig. 16 and Fig.17, shown the result of desired response and the output of PSO algorithm, we can see that the desired response achieve with 500 iteration .note that ,the average different between PSO output and initial solution at null point approximately $15 \mathrm{db}$.

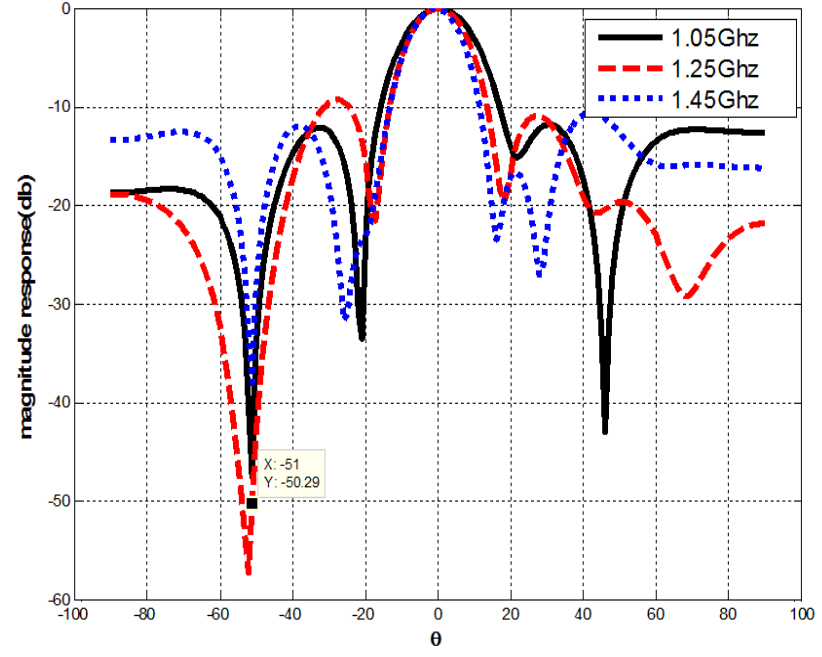

Fig.16: The magnitude response of wideband beamforming with null points at $\theta=-51^{\circ} \mathrm{f}=1.05 \mathrm{GHz}$, $\mathrm{f}=1.25 \mathrm{GHz}, \mathrm{f}=1.45 \mathrm{GHz}$

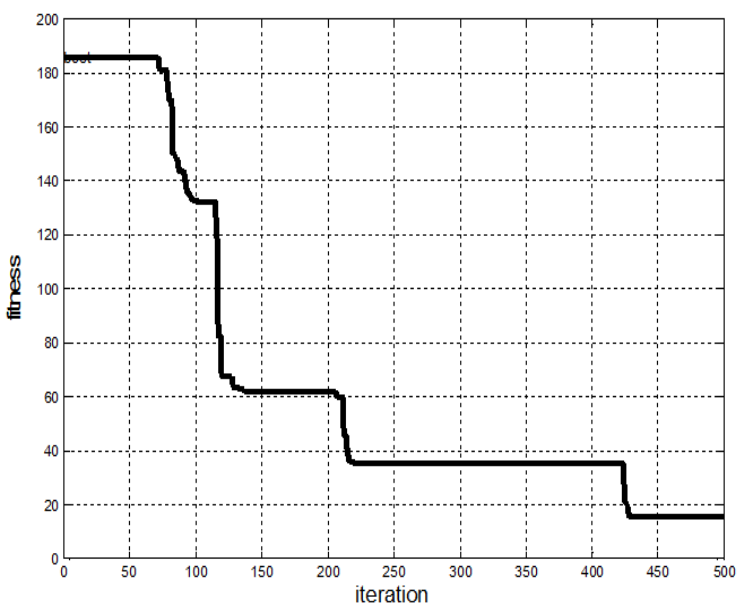

Fig.17: Convergence curve of the fitness function versus the number of iterations

\section{CONCLUSION}

This paper illustrated the use of the particle swarm optimization (PSO) method in design narrowband and wideband beamforming with Taped Delay Lines (FIR Filter) geometry for the purpose of suppressed sidelobes and null placement in certain directions and frequency. The PSO algorithm was successfully used to optimize the weights set for optimal beamformer to exhibit a beamformer response with either suppressed sidelobes null placement in certain directions, or both. In each of these cases the PSO algorithm easily achieved the optimization goal. As an evolutionary algorithm the PSO method will most likely be an increasingly attractive alternative, in the electromagnetic and antennas community, the PSO algorithm is much easier to understand and implement and requires minimum mathematical preprocessing. Beamforming can be designed using new optimization methods such as cuckoo and genetic algorithms, a performance comparison can be done afterwards in order to evaluate each optimization method strengths and weaknesses. Moreover, the application of designing a good null points for beamforming in radar systems, satellite TV and avoiding the jamming on TVs and radar channels. The research in this field is open because of the fact that there is no optimal solution especially for its applications in designing a smart antenna systems. 


\section{REFERENCES}

[1] Godara, Lal C. "Applications of antenna arrays to mobile communications.I. Performance improvement, feasibility, and system considerations." Proceedings of the IEEE 85.7 (1997): 1031-1060.Ding, W. and Marchionini, G. 1997 A Study on Video Browsing Strategies. Technical Report. University of Maryland at College Park.

[2] Godara, Lal C. "Application of antenna arrays to mobile communications. II. Beam-forming and direction-ofarrival considerations." Proceedings of the IEEE 85.8 (1997): 1195-1245.

[3] Takao, Kazuaki, M. Fujita, and T. Nishi. "An adaptive antenna array under directional constraint." IEEE Transactions on Antennas and Propagation 24.5 (1976): 662-669.

[4] Vook, F. W., and R. T. Compton. "Bandwidth performance of linear adaptive arrays with tapped delayline processing." IEEE Transactions on Aerospace and Electronic Systems 28.3 (1992): 901-908.

[5] Frost, Otis Lamont. "An algorithm for linearly constrained adaptive array processing." Proceedings of the IEEE 60.8 (1972): 926-935.

[6] Compton, R. T. "The bandwidth performance of a twoelement adaptive array with tapped delay-line processing." IEEE Transactions on Antennas and Propagation 36.1 (1988): 5-14.

[7] Rodgers, W. E., and R. T. Compton. "Adaptive array bandwidth with tapped delay-line processing." IEEE Transactions on Aerospace and Electronic Systems 1 (1979): 21-28.

[8] Ahmed, K., and R. Evans. "An adaptive array processor with robustness and broad-band capabilities." IEEE transactions on antennas and propagation 32.9 (1984): 944-950.

[9] Er, Meng, and A. N. T. O. N. I. O. Cantoni. "A new approach to the design of broad-band element space antenna array processors." IEEE journal of oceanic engineering 10.3 (1985): 231-240.

[10] Mailloux, Robert J. Phased array antenna handbook. Vol. 2. Boston: Artech House, 2005.
[11] Liu, Wei, and Stephan Weiss. Wideband beamforming: concepts and techniques. Vol. 17. John Wiley \& Sons, 2010.

[12] Robinson, Jacob, and Yahya Rahmat-Samii. "Particle swarm optimization in electromagnetics." IEEE transactions on antennas and propagation 52.2 (2004): 397-407.

[13] Kennedy, James, and R. Eberhart Particle Swarm Optimization. "Ieee int." Conf. on Neural Networks. Vol 4. 1995.

[14] Khawaldeh, Mohammad Kamel, and Dia I. Abu-AlNadi. "Design of wideband beamforming using particle swarm optimization." Systems, Signals and Devices (SSD), 2012 9th International Multi-Conference on IEEE, 2012.

[15] Alchalabi, Alaa Eddin, Mohammed Elsharnoby, and Saed Khawaldeh. "Rightscope: Detecting search campaingns positive and negative queries." Machine Learning and Cybernetics (ICMLC), 2016 International Conference on. IEEE, 2016

[16] Khodier, Majid M., and Christos G. Christodoulou. "Linear array geometry synthesis with minimum sidelobe level and null control using particle swarm optimization." IEEE Transactions on Antennas and Propagation 53.8 (2005): 2674-2679.

[17] Shi, Yuhui. "Particle swarm optimization: developments, applications and resources." evolutionary computation, 2001. Proceedings of the 2001 Congress on. Vol. 1. IEEE, 2001

[18] Eberhart, Russell C., Yuhui Shi, and James Kennedy. "Swarm Intelligence (The Morgan Kaufmann Series in Evolutionary Computation)." (2001).

[19] Trelea, Ioan Cristian. "The particle swarm optimization algorithm: convergence analysis and parameter selection." Information processing letters 85.6 (2003): 317-325.

[20] Zaman, M. A., et al. "Phased Array Synthesis Using Modified Particle Swarm Optimization." Journal of Engineering Science \& Technology Review 4.1 (2011) 